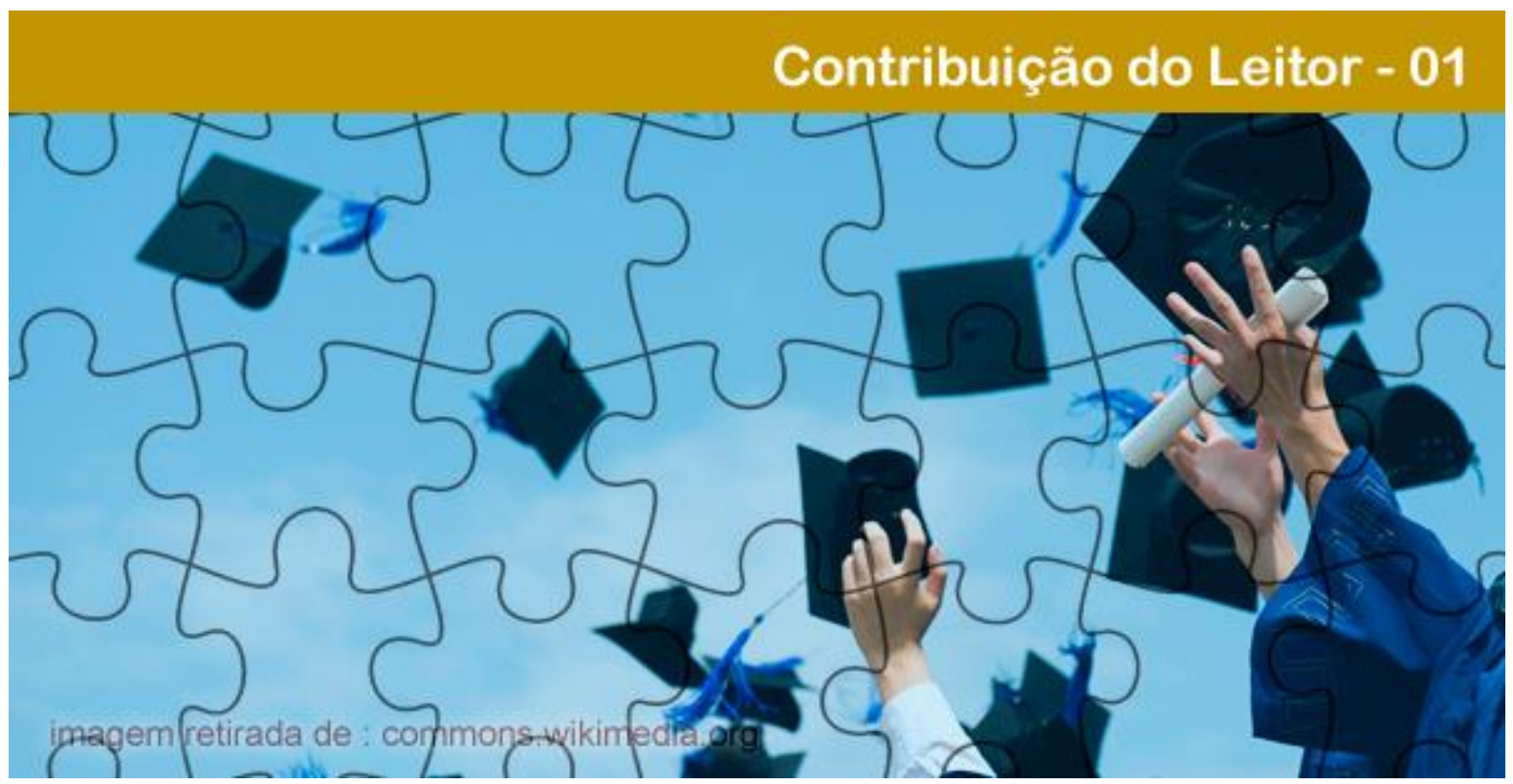

\title{
RESENHA DO LIVRO INOVAÇÃO U 2.0: REINVENTANDO OS PAPÉIS DA UNIVERSIDADE NA ECONOMIA DO CONHECIMENTO (TORNATZKY; RIDEOUT, 2015)
}

\begin{abstract}
Branca Terra
Graduação em Engenharia Civil. Mestrado em Engenharia Civil. Doutorado em Engenharia de Produção pela Universidade Federal do Rio de Janeiro - UFRJ/COPPE (1999). Professora adjunta da Universidade do Estado do Rio de Janeiro, pesquisadora do programa Prociência e líder dos grupos de pesquisa Inovação e Sociedade e Inovação na Gestão Pública. E-mail: brancaterra@gmail.com.
\end{abstract}

\section{Eduardo Pacheco Campos}

Graduação em Administração pela Universidade do Estado do Rio de Janeiro (2014). Tem experiência na área de Administração. E-mail: eduardo.pcampos04@gmail.com.

\section{Jana Almeida Nogueira}

Graduação em direito pela Universidade Candido Mendes. Bolsista de Nível Superior do projeto Qualitec da InovUerj. E-mail: jana.almeida@globo.com.

\section{Leonardo Lehnemann Agostinho Martins}

Graduação em Administração com ênfase em Finanças na Universidade do Estado do Rio de Janeiro. Mestrado em Ciências Contábeis pela Universidade do Estado do Rio de Janeiro. Tem experiência na área de Química, com ênfase em Química Inorgânica, informática (principalmente programação e desenvolvimento de jogos) $e$ administração (ênfase na área financeira).E-mail: lehneman@gmail.com.

Resumo: O livro Innovation U 2.0 Reinventing University Roles in a Knowledge Economy aborda a inovação e o empreendedorismo dentro de doze universidades norte-americanas escolhidas por especialistas como as que mais se destacam na área. Nele estão os estudos de casos dessas universidades onde são apresentados os elementos das suas culturas acadêmicas, as suas principais lideranças, os seus programas curriculares e co-curriculares para promoção do empreendedorismo, políticas, práticas e apoios para a ultrapassagem de fronteiras entre as universidades e o mundo "externo" do setor privado e para a transferência de tecnologia. O objetivo da resenha deste livro é indicar a importância da liderança e da cultura organizacional para o surgimento e a manutenção de políticas e programas que apoiam a inovação dentro das universidades. Além disso, foram selecionados e

\section{POLÊM!CA $\mid$ LABORE (}

Polêmica - Revista Eletrônica da Uerj - Rua São Francisco Xavier, 524, $1^{\circ}$ andar

bloco D, sl.1001 • Tels.: +55 21 2334-4088 / 4087 • http://www.e-publicacoes.uerj.br/index.php/polemica/index http://www.labore.uerj.br • laboreuerj@yahoo.com.br 
descritos alguns programas para que seja percebido como são diversificados e numerosos dentro das universidades presentes nos estudos de caso, e que além de reforçarem a formação empreendedora, eles trazem resultados para a sociedade através de produtos e serviços. Alguns dos centros de pesquisas e laboratórios foram citados, pois eles também estão ligados a produção de inovação e o alto nível do empreendedorismo delas, assim como as políticas e as práticas de transferência de tecnologia apresentadas. As principais conclusões são que a liderança é um fator importante para as universidades que queiram se destacar como produtoras de inovação e, que nessa resenha e no livro resenhado, são encontrados muitos e diversos programas, políticas, e práticas que podem ser reproduzidos por outras universidades.

Palavras-chave: Inovação. Empreendedorismo. Universidade Empreendedora.

\title{
BOOK REVIEW OF INNOVATION U 2.0.: REINVENTING UNIVERSITY ROLES IN A KNOWLEDGE ECONOMY (TORNATZKY; RIDEOUT, 2015)
}

\begin{abstract}
The book Innovation U 2.0 Reinventing University Roles in the Knowledge Economy discusses innovation and entrepreneurship within twelve American universities chosen by experts as the most distinctive in the area. There are case studies of those universities where the elements of their academic cultures are presented, their main leaders, their curriculum and co-curriculum for promotion of entrepreneurship, policies, practices and support for the crossing of borders between universities and the "external" world of the private sector and technology transfer. The objective of the review of this book is to indicate the importance of leadership and organizational culture for the creation and maintenance of policies and programs that support innovation within universities. In addition, some programs are selected and described in order to be perceived how diverse and numerous they are within the universities present in the case studies, and in addition to strengthen the entrepreneurial formation, they also bring results to society through products and services. Some of the research centers and laboratories were also mentioned, because they are linked to the production of innovation and the high level of their entrepreneurship, as well as the policies and presented technology transfer practices. The main conclusions are that leadership is an important factor for universities that want to stand out as producers of innovation and that this review and the reviewed book are found many diverse programs, policies, and practices that can be replicated by other universities.
\end{abstract}

Keywords: Innovation. Entrepreneurship. Innovative Universities.

Este trabalho é uma revisão do livro Innovation U 2.0 ReinventingUniversity Roles in a Knowledge Economy, lançado em 2014, cujo tema é inovação, sob a ótica dos autores e organizadores: Louis G.Tornatzky, PhD e Elaine C. Rideout, PhD. O primeiro autor é professor, gerente, pesquisador e autor de diversos livros e artigos sobre inovação tecnológica, tecnologia, política e empreendedorismo atuando em grandes universidades americanas e em grupos de pesquisa. A segunda autora produziu vários trabalhos na área de empreendedorismo e geração de empresas para o crescimento econômico. O livro também teve a contribuição de diversos especialistas em inovação que trabalhavam em grupos de pesquisa, junto com os autores.

O trabalho é uma continuação do livro Innovation U.: New University Roles in a Knowledge Economy, lançado em 2002, de autoria de Louis G. Tornatzky, também autor do trabalho que aqui está sendo analisado, de Paul G. Waugaman e Dennis O. Gray. O livro de 2002 apresentou doze estudos de caso de universidades americanas de pesquisa, as quais realizavam atividades para promoção de inovação tecnológica e de desenvolvimento

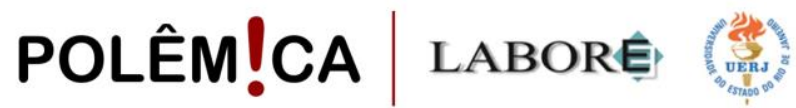

Polêmica - Revista Eletrônica da Uerj - Rua São Francisco Xavier, 524, $1^{\circ}$ andar

bloco D, sl.1001 • Tels.: +55 21 2334-4088/4087• http://www.e-publicacoes.uerj.br/index.php/polemica/index

http://www.labore.uerj.br • laboreuerj@yahoo.com.br 
econômico baseado na tecnologia. ScottDoron, autor do prefácio daquele livro, Tornatzky e Rideoutw (2014) citam:

\begin{abstract}
Ninguém percebeu, há dez anos atrás, o quanto revelador era o livro Innovation $U$. Naquela época, as palavras "desenvolvimento econômico" e "universidades" raramente apareciam juntas. Agora, muitos atores econômicos incluem universidades em suas carteiras de ativos e um grande número de funcionários das universidades interagem propositadamente com suas economias locais. Innovation $U$ não deveria reivindicar o crédito exclusivo dessa mudança, entretanto, pode-se afirmar que contribuiu para a sensibilização de alguns grupos críticos e ajudou a construir o impulso para a elaboração de políticas públicas nessa área.
\end{abstract}

Os objetivos do Innovation $U 2.0$ (p. 254) são: "sintetizar os novos domínios ou as atividades de inovação nas universidades (especialmente de empreendedorismo); observar os papéis de liderança e da cultura para a promoção da inovação na universidade; e observar algumas universidades diferentes que, por diversas razões, não foram apresentadas na amostra dos casos de 2002".

Assim, o livro em análise, Innovation $U$ 2.0, apresenta as doze universidades americanas que mais se destacam na criação de inovação, com impactos econômicos provenientes de startups $^{1}$, selecionadas de uma listagem das 100 universidades de pesquisa que mais receberam suporte da National Science Foundation - NSF no ano fiscal de 2010, além de uma sub-amostra das instituições menores e menos intensivas em pesquisa que, no entanto, tinham reputação como inovadoras. São elas: Arizona State University (ASU); Brigham Young University (BYU); California Institute of Technology (Caltech); Carnegie Mellon University (CMU); Clemson University (Clemson); Georgia Institute of Technology (Georgia Tech); Massachusetts Institute of Technology (MIT); North Carolina State University (NC State); Purdue University (Purdue); Stanford University (Stanford); University of Florida (UF); e a University of Utah.

Nas análises dos casos das doze universidades foram observadas as cinco dimensões (chamados pelos autores de problemas-chave ou oportunidades) relacionadas aos modelos organizacionais e atitudes que as universidades apresentam, em termos de inovação tecnológica. São elas as seguintes:

\footnotetext{
${ }^{1}$ São empresas "[...] em fase embrionária, geralmente no processo de implementação e organização de suas operações. Pode não ter ainda iniciado a comercialização em massa de seus produtos, mas já está a funcionar ou, pelo menos, em processo final de instalação" (TABORDA, 2006).
}

\title{
POLÊM!CA $\mid$ LABORE
}

Polêmica - Revista Eletrônica da Uerj - Rua São Francisco Xavier, 524, $1^{\circ}$ andar 
(1) Cultura da universidade - objetivos e aspirações: estão relacionados os elementos da cultura das universidades - missões, visões e objetivos e estratégias conexas com as atividades de inovação.

(2) Liderança: são apresentados os líderes internos e externos a academia, que atuaram no crescimento da inovação tecnológica dentro das universidades, destacando-se as experiências, funções e os papéis desses atores e suas equipes.

(3) Expansão de fronteiras - empreendedorismo: são descritas as atividades de promoção do empreendedorismo na academia.

(4) Expansão de fronteiras - parceria com a indústria e a comunidade: são apresentadas políticas, práticas e apoios para a transformação da pesquisa em artefato, com expansão das fronteiras acadêmicas para o setor privado.

(5) Expansão de fronteiras - transferência de tecnologia: são apresentadas as estruturas que cuidam da transferência de tecnologia dentro das universidades, onde estão descritas algumas das suas políticas e práticas.

Nos estudos dos casos dessas doze universidades, alguns pontos podem ser evidenciados. Com relação a dimensão (1) anteriormente citada, na $A S U$, um dos seus presidentes, o Dr. Michael Crow, escreveu um documento a respeito do modelo de uma nova universidade denominado New American University. Esse documento fortaleceu a inovação e o empreendedorismo, além de incluir a criação de departamentos transdisciplinares e políticas que motivam os alunos e professores para atuarem no processo de inovação, como por exemplo, o Arizona Technology Enterprises que é a unidade de transferência de tecnologia da $A S U$ e o Venture Catalyst, que atua comostart-upaccelerator, conectando a universidade às empresas locais e nacionais.

Já a $B Y U$ oferece um ensino que possibilita os egressos atuarem em ciências, inovação e negócios, compreendendo os processos práticos e o ambiente técnico. Outro exemplo é a Caltech, cuja missão é ampliar o conhecimento da humanidade e beneficiar a sociedade através da pesquisa integrada com a educação. Enfatiza a pesquisa de problemas desafiadores na ciência e tecnologia, em uma atmosfera interdisciplinar, universitária, educando os alunos para se tornarem pessoas criativas na sociedade, considerando prioritárias as áreas de energia; terra e meio ambiente; ciência médica; ciência da informação; materiais avançados e nanociência e o Universo.

\section{POLÊM!CA $\mid$ LABORE}


Com relação à dimensão (2), como exemplos de líderes, foram citados presidentes, vice-presidentes, reitores, diretores, Corporative Executive Officers - CEOs, fundadores, professores, administradores e governadores que atuaram de forma significativa, demonstrando a vontade política de valorizar o processo de inovação e a formação empreendedora na academia. Na Caltech, CMU, Georgia Tech, MIT, Purdue, Stanford e Universityof Utah houve sucessões de presidentes que permitiram e incentivaram a inovação dentro da universidade. Foram também mostrados alguns exemplos de liderança por parte dos professores, nas universidades, $C M U, U F, N C$ State, MIT e Stanford. Alguns desses líderes já haviam trabalhado em outras universidades que possuíam notória reputação na realização de pesquisas, inovação e empreendedorismo e em escritórios de transferência de tecnologia, e outros já haviam criado startups. A seguir, serão destacados alguns cargos ocupados pelos líderes, parte do trabalho realizado por eles e os seus resultados: 1) Um dos presidentes da $A S U$ contribuiu para a criação de vários centros de pesquisa e institutos, entre eles o Columbia Earth Institute, o Center for Environmental Researchand Conservation, e o Center for Science Policyand Outcomes em Washington. Além disso, um dos reitores criou o Knight Center for Digital Media Entrepreneur ship, o Cronkite Institute for High School Journalism, e o New Media Innovation Lab. 2) Na BYU um reitor, que tinha sua formação em engenharia mecânica, com especialização em design, supervisionou 250 projetos de design da graduação e pós-graduação, com foco na inovação. 3) Na Caltech um dos seus presidentes foi coautor num artigo importante sobre o impacto da pesquisa científica na sociedade. 4) Um dos presidentes da $C M U$, no início do século 20, liderou a fundação e o crescimento da Carnegie Technical Schools (de 1903 até 1912), e do Carnegie Institute of Technology (até 1922). Outros presidentes fundaram laboratórios de pesquisa para metais, carvão, química e física, o Computation Center, o Robotics Institute, e criaram um programa de transferência de tecnologia. Eles também fizeram com que a universidade interagisse com o Pittsburgh Technology Center e o Regional Economic Revitalization Initiative. 5) $\mathrm{Na}$ Clemson presidentes, vice-presidentes e reitores gerenciaram o orçamento e o processo de reorganização da universidade e enfatizaram algumas áreas que permitiram o agrupamento das disciplinas de modo a se encaixarem com os clusters econômicos do estado de South Carolina. 6) $\mathrm{Na} U F$ o presidente, o vice-presidente e diretores expandiram os centros de pesquisas ligados às indústrias, criando cargos focados em tecnologia, promovendo parcerias

\section{POLÊM!CA $\mid$ LABORE}


de pesquisa com outras instituições e com várias iniciativas de tecnologia do estado da Florida, além da reorganização do Office of Technology Licensing (OTL), incluindo novos serviços e atividades fazendo com que a universidade assumisse uma posição de liderança nacional no desempenho da transferência de tecnologia. 7) Na Georgia Tech os presidentes aumentaram o financiamento de pesquisas, o número dos centros de pesquisas e institutos, a qualidade do ensino da graduação e a participação dos estudantes em pesquisas, as atividades de transferência de tecnologia, além de fomentar a atuação junto ao Georgia Research Alliance. 8) No MIT os presidentes, vice-presidentes e professores atuaram na restruturação do currículo da graduação e pós-graduação, focando no processo de inovação e na formação empreendedora, fundaram o David H. Koch Institute for Integrative Cancer Researche, apoiaram o Kendall Square, local onde podem ser instaladas empresas do ramo das ciências da vida e da biotecnologia, colaborando para que o MIT se tornasse líder em pesquisa e desenvolvimento focado na defesa nacional, desenvolvendo novos produtos e patentes, e criação de empresas. 9) Na NC State os governadores do estado da North Carolina, além de um presidente e um executivo de um banco, criaram o Research Triangle Development Councile, o Research Triangle Committee, dando apoio à criação do Research Triangle Park, através de campanha para obtenção de financiamento. 10) Na Purdue foram destacados 10 presidentes, sendo que um deles criou a School of Aeronautics e a Purdue Research Foundation, outro aumentou dez vezes o orçamento em pesquisa e a seguir outro criou novas escolas de Industrial Engineering, Materials Engineering, Technology, o Veterinary Medicine e o Purdue Research Park. 11) Em Stanford os presidentes ajudaram na criação do Stanford Research Park e do Stanford Office of Technology Licensing, também promovendo a criação de vários centros e programas de pesquisa que alcançaram destaque nacional. 12) $\mathrm{Na}$ University of Utah um dos presidentes organizou uma campanha comunitária de educação com ênfase nos benefícios de uma universidade intensiva em pesquisa, o que resultou em um grande número de empresas spin-offs provenientes das pesquisas da universidade e o crescimento da carteira de pesquisa patrocinada, ocasionando um crescimento significativo nos rankings nacionais de universidades.

Com relação à dimensão 3 , pode-se exemplificar na $A S U$ a criação de cursos e competições voltadas para o empreendedorismo social, tais como o estudo aprofundado sobre Empreendedorismo Social e o Edson Student Entrepreneur Initiative. No MIT os exemplos

\section{POLÊM!CA $\mid$ LABORE}


são o escritório do programa de ensino de graduação $D$-Lab e o GlobalHealthLab, com foco em inovação e empreendedorismo. Para a resolução de problemas locais pode-se exemplificar na ASU o Sun Devil Entrepreneur ship Network e o Spirit of Enterprise Center, na CMU o CREATE Lab, na Georgia Tech, o AMAC (Accessibility Solutions), o Health IT Outreach Partnership e o Minority Business Development Agency (MBDA) Business Center-Atlanta e no MIT, o MITV enture Ships Club.

Ainda na dimensão 3, pode-se citar atividades interdisciplinares que as universidades realizam, algumas delas são os programas de desenvolvimento de produtos e as atividades de consultoria, aproximando a universidade da comunidade. Por exemplo, o curso Integrated Product/Service Development (IPD) da CMU e o Integrated Technology Venture Program (ITV) da UF, o TI:GER (Technological Innovation: Generating Economic Results) realizado pela Georgia Tech, e o Biomedical Enterprise Program (BEP) do MIT e a Juelsgaard Clinic da Stanford onde os estudantes de direito analisam questões que podem facilitar ou dificultar a inovação e o empreendedorismo.

O MIT tem o Founders Journey Course que é oferecido aos alunos de graduação da escola de engenharia, ele é um curso de desenvolvimento de produtos e design. O Gordon Engineering Leadership Program também é oferecido para eles, nele é promovida a inovação e liderança, incluindo a inovação dentro de empresas estabelecidas. E o curso Medical Device Design onde alunos da graduação e pós-graduação aplicam fundamentos de engenharia mecânica e elétrica para projetarem novos dispositivos para médicos que atendam às necessidades clínicas.

Na Caltech, no curso Entrepreneurial Development E102, é avaliada a viabilidade das tecnologias ainda não comercializadas da Calteche, os alunos desenvolvem um caso de negócios, e no Management of Technology E / ME 103 equipes de alunos avaliam a tecnologia de uma empresa ou realizam uma consultoria e auditoria das capacidades inovadoras da empresa.

$\mathrm{Na}$ maioria das universidades analisadas existem os clubes de estudantes empreendedores, e eventos tais como competições de planos de negócios e palestras com empreendedores. Também oferecem atividades de assistências para os alunos e professores empreendedores, como mentoring; cursos voltados para a criação e o desenvolvimento de

\section{POLÊM!CA $\mid$ LABORE}


produtos e gerenciamento do negócio; e incubadoras, para os inventores exercerem as atividades do negócio e realizar em networking.

Na Stanford, por exemplo, o Center for Entrepreneurial Studies e o Graduate School of Business Entrepreneur Ship Club ajudam aos alunos e professores a realizarem networking. Sendo que o último organiza apresentações, conversas e outros eventos.

$\mathrm{Na}$ dimensão 4, exemplifica-se os vários centros de pesquisa da $A S U$ que recebem suporte financeiro de parceiros de negócio em um formato de consórcio, sendo que a maioria deles tem como base a Fult on School. Estão incluídos: o Power Systems Engineering Research Center, o Water and Environmental Technology (WET Center), o Center for Embedded Systems (CES), o Net-Centrics System and Software (Net Centric). Na BYU existem mais de 50 centros e institutos de pesquisa além de também participar de parcerias através do Industry-University Cooperative Research Centers (I/UCRC) da NSF com universidades maiores e mais intensivas em pesquisas. Na Caltech há também muitos centros de pesquisas e parcerias com outros centros, entre eles estão: o Joint Center for Artificial Photosynthesis; o Light-Material Interactions in Energy Conversion; Ronald and Maxine Linde Center for Global Environmental Science; Terrestrial Hazard Observation and Reporting (THOR); e oMaterials and Process Simulation Center (MSC).

O MIT também participa de vários programas de centros operados pela National Science Foundation (NSF). Entre eles está o Science and Technology Center in Emergent Behaviors of Integrated Cellular Systems, em colaboração com a Georgia Tech e a University of Illinois, além de possuir parceria com um NSF Science and Technology Center focado em Science of Information, e o Science and Technology Center on Microbial Oceanography liderado pela University of Hawaii. É parceiro também do Science and Technology Center for Biophonics, liderado pela University of California, Davis, e opera o NSF Materials Center on Materials Science and Engineering.

Os centros de inovação e empreendedorismo das universidades analisadas, em geral, estão voltados para áreas como tecnologia da informação, energia, saúde, indústria automobilística, exploração espacial, mudanças climáticas, materiais, tecnologias para deficientes. Vários deles buscam atender as necessidades da comunidade e das empresas nas regiões onde estão inseridas. A Purdue, por exemplo, desenvolve o Technical Assistance Program (TAP), que tem por missão (pp. 199-200) "fazer avançar a prosperidade econômica,

\section{POLÊM!CA $\mid$ LABORE}


saúde e qualidade de vida em Indiana" e o College of Technology comprometido em atender as necessidades de pessoal e na resolução de problemas dos negócios e da indústria de Indiana.

Entretanto, a dimensão 4 não se restringe a apenas os centros e laboratórios. No Stanford Research Park estão localizadas empresas com interesses tecnológicos complementares à universidade. Elas geralmente são empresas tecnológicas que tem conexões com os programas da instituição. Essa experiência deu origem ao Industrial Affiliates Programs. Existem mais de 50 Industrial Affiliates Programs em operação, com oportunidades de as empresas interagirem com os departamentos de ensino acadêmico, centros, fóruns ou institutos. Elas podem dar suporte às pesquisas, participar de reuniões e eventos e receber cópias de relatórios.

A Georgia Tech criou o Georgia Research Alliance (GRA), do qual a universidade é sócia. Ela faz investimentos estratégicos em pessoas e instalações com objetivo de construir centros de excelência em pesquisa nas universidades localizadas em Georgia. A GRA também fomenta parcerias de pesquisa entre as instituições participantes.

O Industrial Liaison Program (ILP) do MIT também está na dimensão 4. Para cada empresa membro dele é atribuído um Industrial Liaison Officer (ILO), que é um facilitador dos compromissos da empresa com membros do corpo docente e a especialidade de um dos centros. Os ILOs podem organizar encontros que envolvam o pessoal da companhia e membros da comunidade de pesquisa do MIT. Também estão disponíveis eventos e conferências para as empresas associadas.

A dimensão 5 exemplifica que na Clemson, a função de transferência de tecnologia e as atividades associados estão organizacionalmente localizadas no Clemson University Research Foundation (CURF) e atua na divulgação do patenteamento, e nas atividades de licenciamento, realizando intermediações por membros do CURF Board para a facilitação da descoberta de novos produtos e a chegada deles ao mercado.

$\mathrm{Na}$ Stanford, o Office of Technology Licensing (OTL) trata da transferência de tecnologia, atuando nas atividades de disclosing e licenciamento, organizando a distribuição dos Royalties entre o inventor, a escola do inventor, e o departamento do inventor para que seja incentivada a transferência de tecnologia.

\section{POLÊM!CA $\mid$ LABORE}


$\mathrm{Na}$ University of Utah, o Technology and Venture Commercialization (TVC) office cuida da transferência de tecnologia, oferecendo orientação processual e estratégica que inclui políticas articuladas, procedimentos, formulários e aconselhamento pessoal; auxiliando na comercialização de intelectual property via licenciamento, bem como ajuda no lançamento de start-ups baseadas na inovação, disponibiliza inclusive guias on-line para seus clientes (InventorsGuideeStartup Guide). O TVC também realiza eventos e serviços abertos a alunos, professores e a grande parte da comunidade de Utah, oferecendo orientação para os inventores e aproximação entre eles e a indústria, workshops, seminários, palestras, realização de pitches e uma oportunidade de fazer parte de uma nova abordagem para acelerar a avaliação, habilitação de negócios e comercialização das invenções em estágio inicial.

Finalmente, no Sumário e Recomendações, os autores indicam que os estudos de caso demonstraram (p. 245): (1) que há universidades que são comprovadamente mais "inovadoras" do que a maioria de seus pares (mesmo aquelas que estão mais ou menos no mesmo nível dos gastos com pesquisas); (2) que existem políticas, práticas e padrões de comportamento que podem ter uma relação causal com a "capacidade de inovação;" (3) que políticas e práticas ligadas à inovação apresentadas são ínfimas se comparadas a todo o contexto americano de inovação; (4) no entanto, as práticas e políticas que são apresentadas são promissoras, bem como adaptáveis, e as outras universidades podem copia-las. Também citam sugestões do que as universidades devem considerar para serem empreendedoras. São as seguintes (pp. 253-254): incentivo a uma orientação externa para o setor privado, contratação de talentos, construção de uma cultura empreendedora; incentivo à prática do que é ensinado, identificar as oportunidades nas iniciativas focadas em inovação realizadas pelo governo federal e estadual, adotar orientação baseada em resultados, e estabelecer orçamento estratégico e tático voltado à inovação e a formação empreendedora.

Ao final do livro, os autores enfatizam que para se fazer uma universidade atingir o nível de desempenho compatível com a inovação apresentada nos doze exemplos analisados, a liderança deve ser tratada como um fator crítico.

\section{POLÊM!CA $\mid$ LABORE}




\section{Referências}

TABORDA, Ana. O que é uma start up? 2006. Disponível em:

<http://www.gesentrepreneur.com/pdf/o_que_e_uma_start_up.pdf>. Acesso em: 13 set. 2016.

TORNATZKY, Louis G.; RIDEOUT, Elaine C. Innovation U 2.0: Reinventing University Roles in a Knowledge Economy, 2014. Disponível em: 〈www.Innovation-U.com>. Acesso em: 21 set. 2015.

Recebido em: 25/05/2016.

Aceito em: 15/09/2016.

\section{POLÊM!CA $\mid$ LABORE}

Polêmica - Revista Eletrônica da Uerj - Rua São Francisco Xavier, 524, $1^{\circ}$ andar bloco D, sl.1001 • Tels.: +55 21 2334-4088/4087 • http://www.e-publicacoes.uerj.br/index.php/polemica/index http://www.labore.uerj.br • laboreuerj@yahoo.com.br 